\title{
A COMPARISON OF THE HINGE AND NEAR-HINGE FLOW FIELDS OF THE ST JUDE MEDICAL HEMODYNAMIC PLUS AND REGENT BILEAFLET MECHANICAL HEART VALVES
}

Jeffrey T. Ellis, $\mathrm{PhD}^{\mathrm{a}}$

Ajit P. Yoganathan, $\mathrm{PhD}^{\mathrm{b}}$
Objective: The most widely implanted prosthetic valves are the mechanical bileaflets, most of which have good forward flow hemodynamics. However, recent clinical experiences illustrate the importance of understanding the flow structures generated within the hinge. The purpose of this study was to evaluate the hinge-flow dynamics of two new variations of a 17-mm St Jude Medical bileaflet valve: the Hemodynamic Plus and the Regent (St Jude Medical, Inc, St Paul, Minn). Methods: Clinical quality reproductions of the valves were manufactured with clear housings. Laser Doppler velocimetry velocity and turbulent shear stress measurements were conducted within the hinge and thumbnail regions of the valves. Results: In the 17-mm Hemodynamic Plus hinge, a rotating flow structure developed in the inflow pocket during forward flow. During systole, velocities through the hinge pocket reached $0.70 \mathrm{~m} / \mathrm{s}$, and the turbulent shear stress reached $1000 \mathrm{dynes} / \mathrm{cm}^{2}$. In the thumbnail, forward flow velocities ranged from $1.4 \mathrm{~m} / \mathrm{s}$ to $1.7 \mathrm{~m} / \mathrm{s}$. In the $17-\mathrm{mm}$ Regent hinge, a rotating flow structure partially developed in the inflow pocket during forward flow. During systole, velocities through the hinge pocket reached $0.75 \mathrm{~m} / \mathrm{s}$, and the turbulent shear stress reached 1300 dynes $/ \mathrm{cm}^{2}$. In the thumbnail, forward flow velocities ranged from $\mathbf{1 . 0}$ $\mathrm{m} / \mathrm{s}$ to $1.3 \mathrm{~m} / \mathrm{s}$. Conclusions: The active leaflet motion through the St Jude Medical hinge creates a washout pattern that restricts the persistence of stagnation zones and thus may be a contributing factor to its successful clinical performance. The hinge and thumbnail flow dynamics of the 17-mm Regent valve are at least equivalent to, and possibly superior to, those of the 17-mm Hemodynamic Plus valve. (J Thorac Cardiovasc Surg 2000;119:83-93)

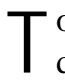
oday the most widely implanted prosthetic valve design is the mechanical bileaflet, ${ }^{1}$ the proper functioning of which requires the use of a hinge mechanism. However, the implications of this requirement have received comparatively little attention given that the hinge can directly affect valve durability, functionality,

From the School of Mechanical Engineering ${ }^{\mathrm{a}}$ and the School of Biomedical Engineering, ${ }^{\text {b }}$ Georgia Institute of Technology, Atlanta, Ga.

Supported by a research contract with St Jude Medical, Inc. Partial funding was also provided by a grant through the Georgia Affiliate of the American Heart Association.

Received for publication April 21, 1999; revisions requested Aug 8, 1999; revisions received Sept 10, 1999; accepted for publication Sept 16, 1999.

Address for reprints: Ajit P. Yoganathan, PhD, Associate Chair, School of Biomedical Engineering, Georgia Institute of Technology, 654 Cherry St, Atlanta, GA 30332-0535.

Copyright $(\odot) 2000$ by Mosby, Inc.

$0022-5223 / 2000 \$ 12.00+0 \quad \mathbf{1 2 / 1 / 1 0 3 0 1 6}$ and potential thrombogenicity. ${ }^{2}$ Recent studies of the unacceptably high thrombosis rates of the Medtronic Parallel (MP) bileaflet mechanical heart valve (Medtronic, Inc, Minneapolis, Minn) have provided strong evidence attesting to the importance of hinge design in the overall clinical performance of a bileaflet valve. ${ }^{3-5}$ In these studies the flow through the MP hinge was found to be dominated by unsteadiness, vortical structures, and multiple zones of stagnation, especially within the inflow pocket. These findings were supported by limited clinical explant data that illustrated thrombus formation in the inflow pocket.

In contrast to the performance of the MP valve, the St Jude Medical (SJM) valve (St Jude Medical, Inc, St Paul, Minn) has presented very low long-term rates of thrombogenicity and valve-related events. ${ }^{6,7}$ The SJM standard bileaflet mechanical heart valve was introduced for clinical use in the United States in 1977. The hinge design of this valve consists of a semicircular pro- 


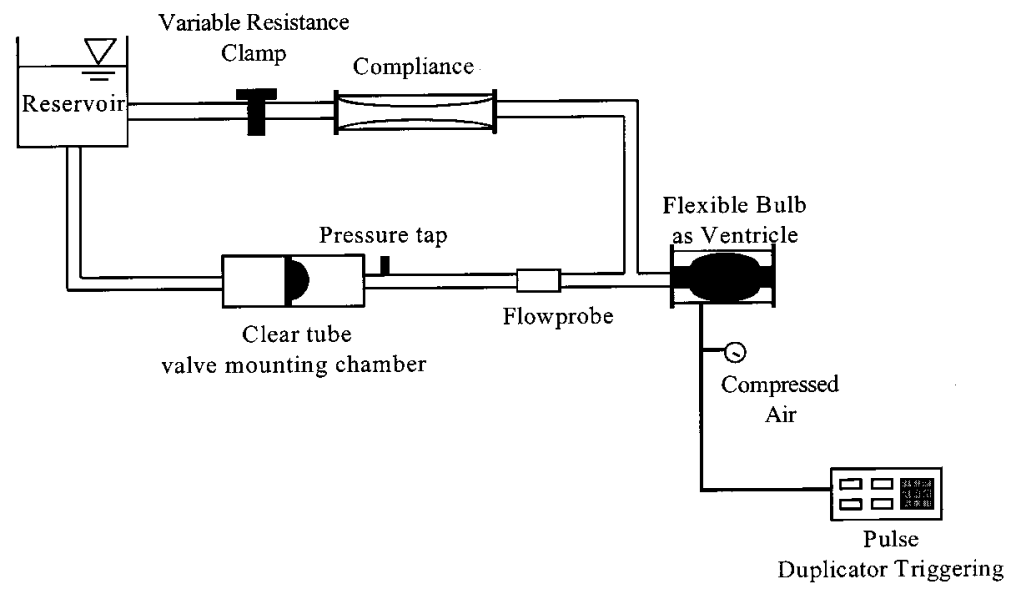

Fig 1. Pulsatile flow loop used for mitral hinge flow studies.

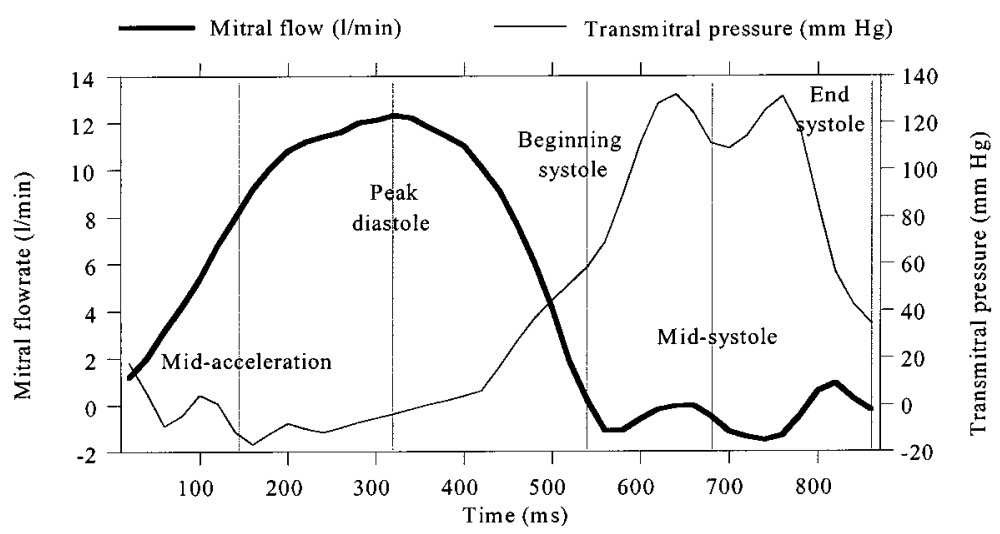

Fig 2. Representative mitral flow and transmitral pressure waveforms.

jection on the leaflet, called the ear, which mates to a similarly shaped recess in the valve housing. The four hinge recesses are machined into two protrusions called pivot guards that project from the inflow side of the valve. Recently the SJM standard series has undergone further innovation. The Hemodynamic Plus (HP) series was introduced in 1993 and included a sewing cuff modification that achieved a larger effective orifice area compared with that of the SJM standard valve, with an equivalent valve anulus diameter. The hemodynamic performance of the SJM HP series is equivalent to that of the next size larger SJM standard. The SJM Regent series, now in clinical trials, includes an increase in the orifice dimensions over the HP series by modifying the outside geometry of the orifice housing to preserve mechanical integrity.

The purpose of this study was to evaluate and compare the hinge flow dynamics of the SJM HP and
Regent size $17 \mathrm{~mm}$ bileaflet mechanical heart valves. These studies were conducted with the primary goal of obtaining an improved understanding of how the SJM hinge design has contributed to its comparatively successful clinical record. In addition, these studies provide a direct comparison that will be useful in assessing any significant differences between the hinge flow dynamics of the HP and Regent series.

\section{Methods}

The studies were conducted in the Georgia Tech left heart pulse duplicator. St Jude Medical provided 17-mm HP and Regent valve models with clear housings and clinical quality dimensions and tolerances. The valve models were mounted in the mitral position, as shown in Fig 1. Fig 2 shows representative mitral flow and transmitral pressure waveforms obtained during the studies. Flow rates were measured with an ultrasonic flowmeter (model T108; Transonic Systems Inc, Ithaca, NY), and relevant pressures were measured with 

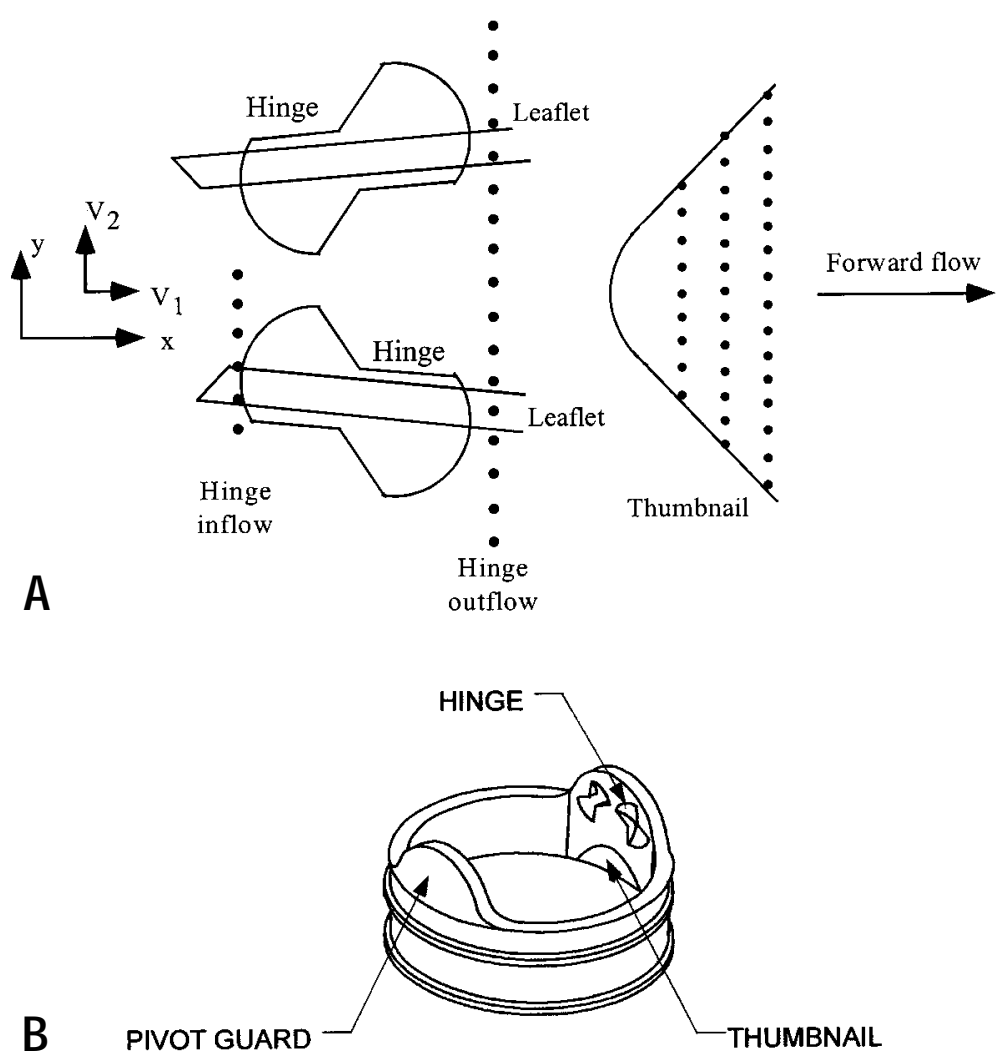

Fig 3. A, Representative top view of LDV near-hinge regions. B, Perspective view of hinge and thumbnail.

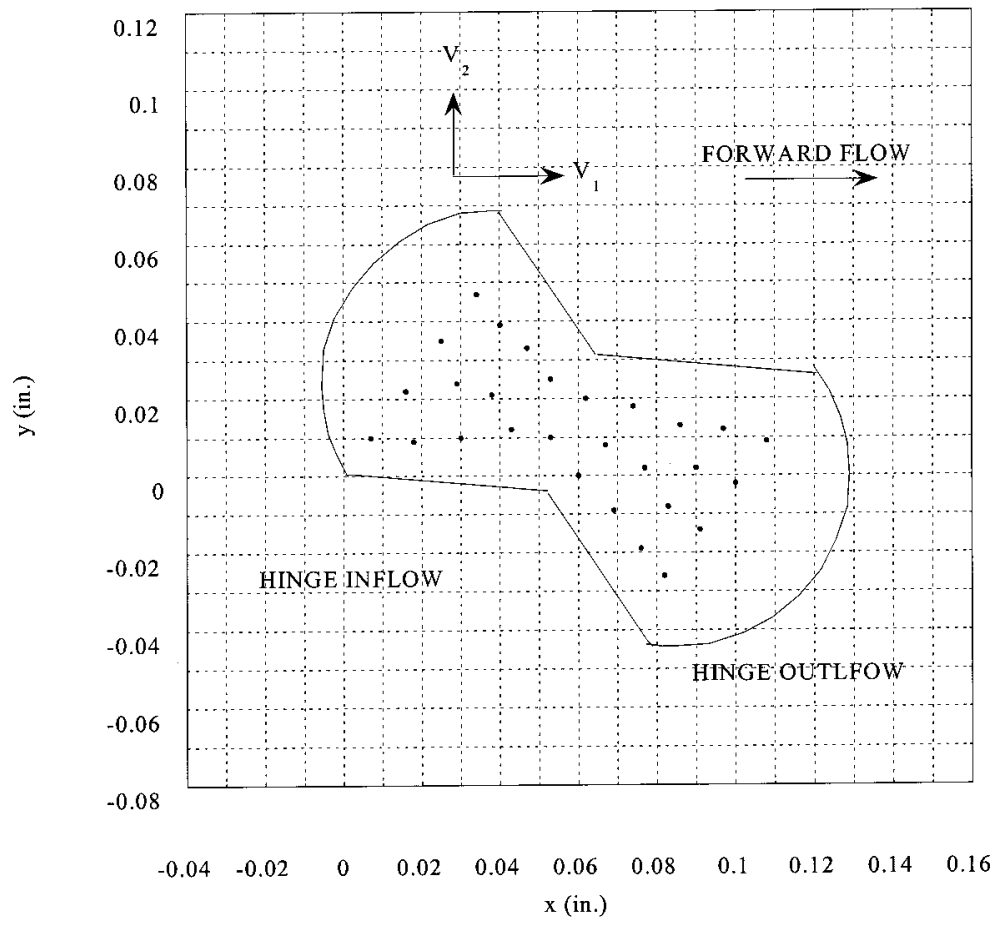

Fig 4. Representative top view of LDV hinge flow sites. 


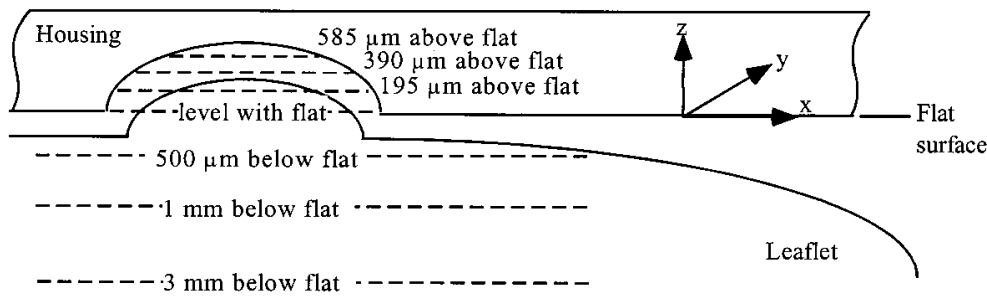

Fig 5. Elevated view of LDV measurement sites.

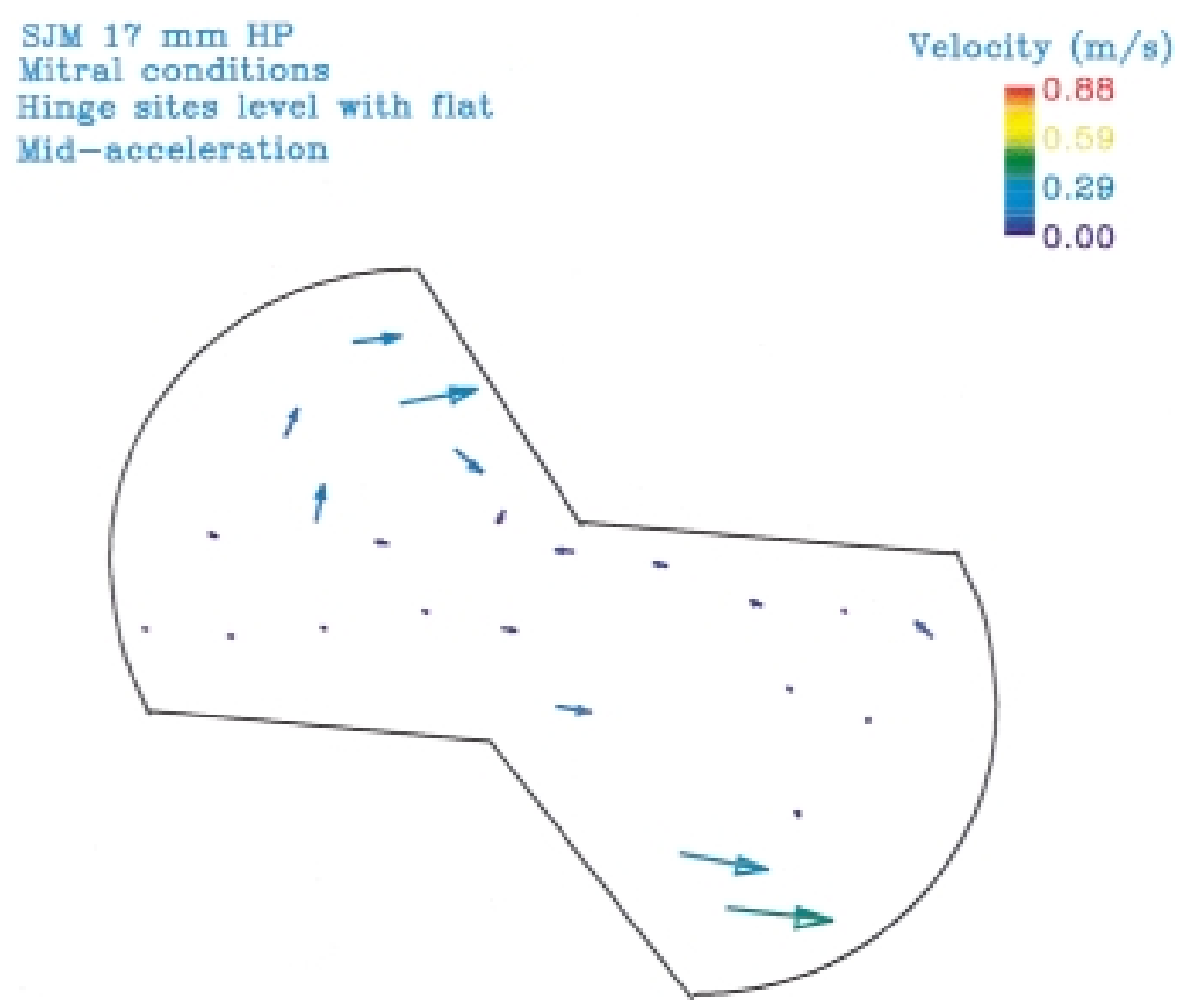

Fig 6. SJM 17-mm HP hinge flow field at mid-acceleration, level with flat housing surface.

a physiologic pressure transducer (model P23-ID; GouldStatham Instruments, Los Angeles, Calif). A three-component, fiberoptic, laser Doppler velocimetry (LDV) system (Aerometrics Incorporated, Sunnyvale, Calif) with a $140-\mu \mathrm{m}$ sample volume resolution was used to obtain two-component, coincident velocity measurements within the hinge and nearhinge regions of the valves. Further details of this system have been published previously. ${ }^{3}$

Fig 3, A, shows a top view of the near-hinge regions of interest. These regions included a plane across the inflow edge of the hinge recesses, a plane across the outflow edge, and three planes within the thumbnail. The thumbnail is a region of slight expansion that is machined into the housing of the SJM valve and is shown in the perspective view of Fig
$3, B$. Figs 4 and 5 show top and elevated views of the measurement sites within the hinges.

At each measurement site, the pulsatile data were divided into bin widths of $20 \mathrm{~ms}$. This width was chosen because it is representative of the time over which changes in blood element morphology can occur in a shear field. ${ }^{8,9}$ The maximum or principal values of the turbulent shear stresses (TSSs) were determined according to the formulation published by Baldwin and colleagues. ${ }^{10}$ The TSS values are of particular interest because they characterize a momentum exchange that may lead to a transfer of forces through formed blood elements, resulting in membrane damage or distortion. This increased mechanical loading can precipitate the elevated release of markers associated with blood element damage, 
Volume 119, Number 1

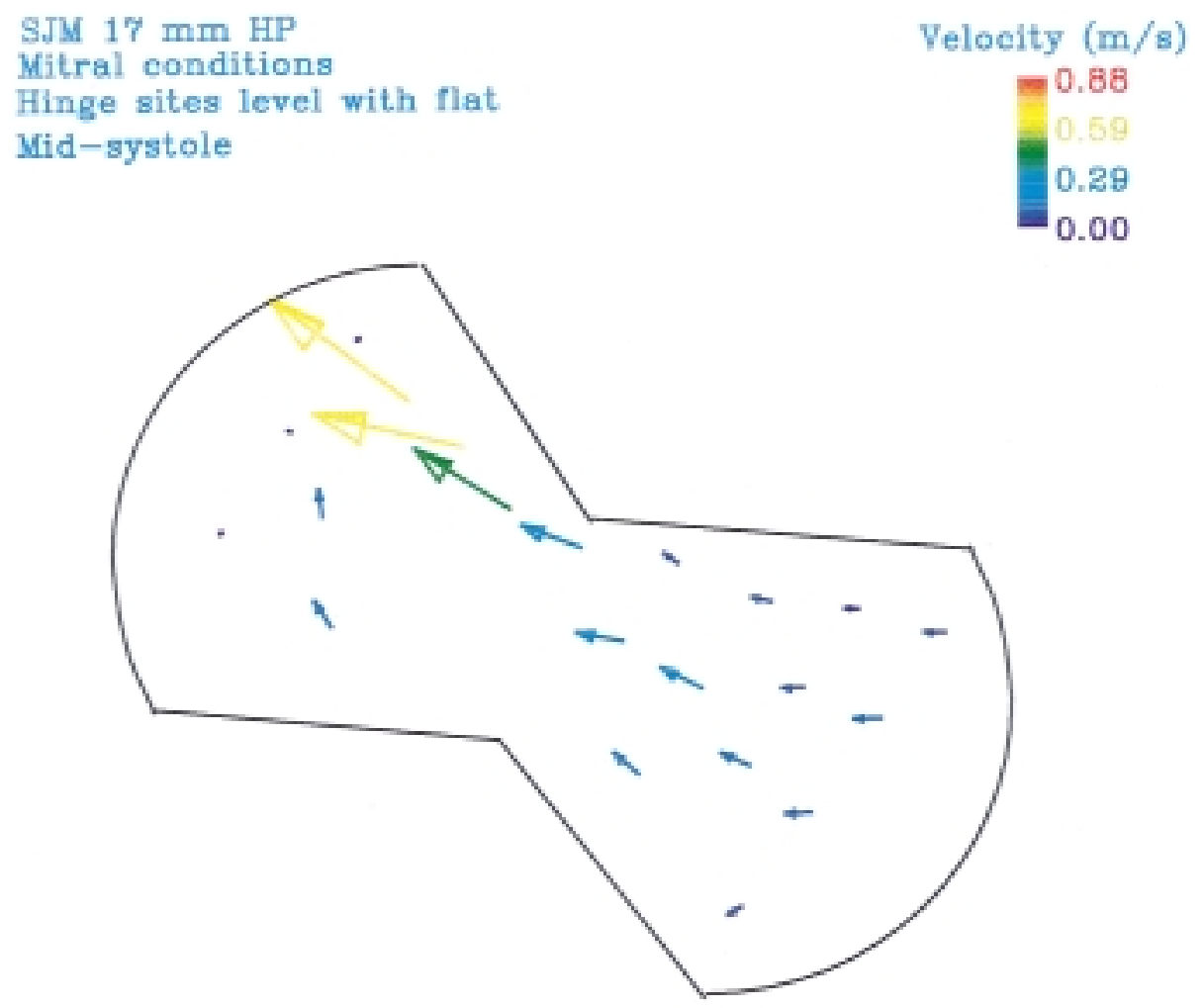

Fig 7. SJM 17-mm HP hinge flow field at mid-systole, level with flat housing surface.

particularly when the characteristic length scales of the flows are on the order of blood element dimensions. . $^{3,10,11}$

\section{Results}

The results are presented in the form of color pictures that illustrate the flow fields at specific instances during the cardiac cycle. The arrows in the figures point in the direction of the mean velocity vectors and are color coded by the velocity magnitudes given in the figure legends. The orientation is as given in Figs 3, A, and 4.

SJM 17-mm HP hinge flow fields. Fig 6 shows the velocity field at mid-acceleration inside the hinge region of the 17-mm HP valve, level with the flat housing surface. During this phase, a rotating flow structure developed in the inflow hinge pocket with velocities ranging from $0.15 \mathrm{~m} / \mathrm{s}$ to $0.30 \mathrm{~m} / \mathrm{s}$ and TSS level reaching $200 \mathrm{dynes} / \mathrm{cm}^{2}$. Slightly higher velocities of up to $0.40 \mathrm{~m} / \mathrm{s}$ were observed near the outer edge of the outflow hinge pocket. The mid-acceleration velocity fields were mostly stagnant at the remaining elevations.

At peak diastole, the rotating flow structure that developed in the inflow hinge pocket was still present at the level of the flat housing surface. In the rotating structure the velocities ranged from $0.18 \mathrm{~m} / \mathrm{s}$ to 0.40 $\mathrm{m} / \mathrm{s}$, and the TSS level reached 100 dynes $/ \mathrm{cm}^{2}$. The peak diastolic velocity fields were mostly stagnant at the remaining elevations, with velocities of less than $0.10 \mathrm{~m} / \mathrm{s}$.

Fig 7 shows the velocity field at mid-systole, level with the flat housing surface. The rotating flow structure that was present at mid-acceleration and peak diastole was abolished during mid-systole as the leakage jet flowed through the hinge. In the outflow hinge pocket, the vectors were observed to flow toward and along the inside surface of the leaflet ear, with velocities reaching $0.30 \mathrm{~m} / \mathrm{s}$. In the inflow hinge pocket, the leakage jet was squeezed from between the hinge wall and the inside surface of the leaflet ear. Velocities reached 0.70 $\mathrm{m} / \mathrm{s}$, and the TSS level reached 2900 dynes $/ \mathrm{cm}^{2}$ in this region. The velocity fields inside the hinge region at the $195-\mu \mathrm{m}$ and $390-\mu \mathrm{m}$ elevations were similar to those observed at the level of the flat housing surface. At these elevations, leakage velocities reached $1.3 \mathrm{~m} / \mathrm{s}$, and the TSS level reached 1700 dynes $/ \mathrm{cm}^{2}$.

SJM 17-mm HP hinge inflow, outflow, and thumbnail flow fields. At mid-acceleration, the central jet was skewed at each elevation, with recirculation regions apparent at the jet boundaries. The maximum 


\section{SJM $17 \mathrm{~mm}$ HP Nitral conditions Hinge inflow, outflow, and thumbnail $1 \mathrm{~mm}$ below flat Peak diastole}
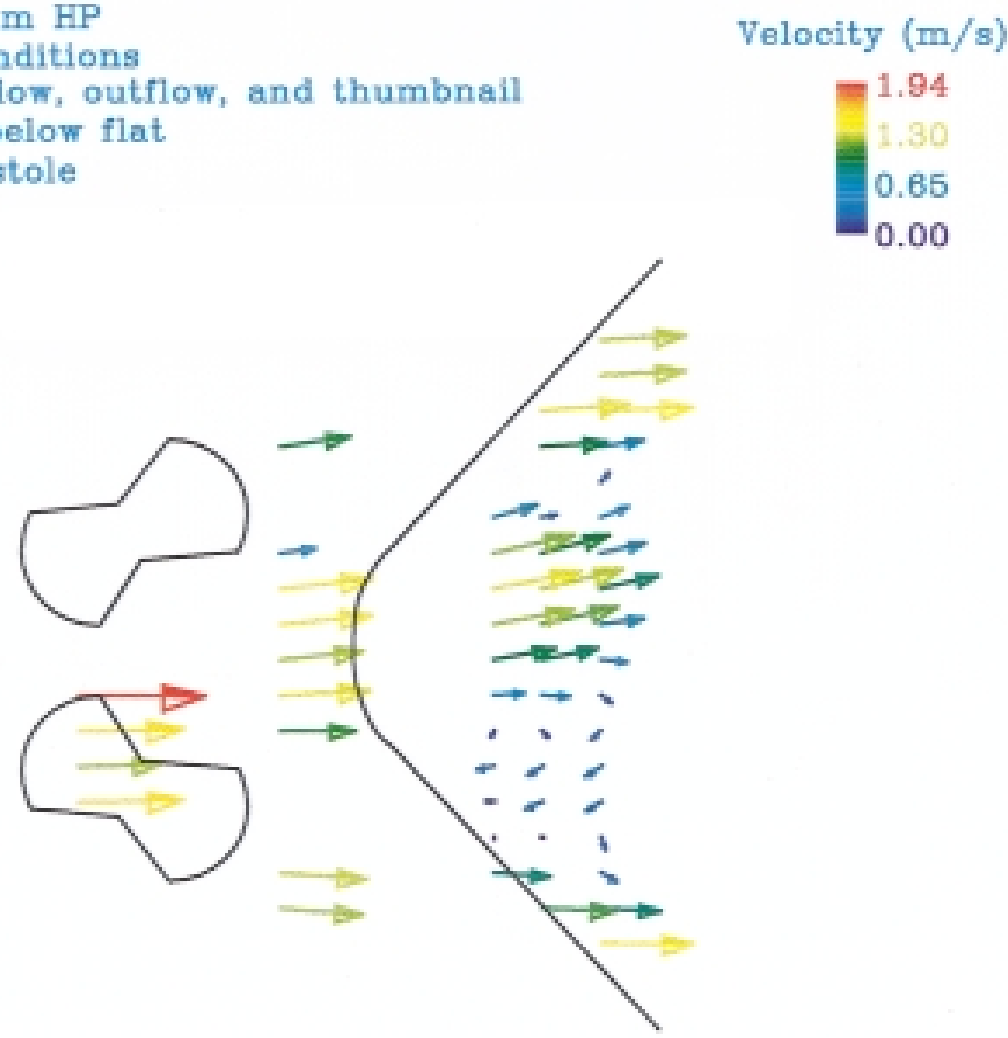

Fig 8. SJM 17-mm HP hinge inflow, outflow, and thumbnail flow field at peak diastole, $1 \mathrm{~mm}$ below flat housing surface.

jet velocity was $1.6 \mathrm{~m} / \mathrm{s}$, as measured at the $3-\mathrm{mm}$ elevation.

By peak diastole, a small area of forward flow was present at the $500-\mu \mathrm{m}$ elevation in the thumbnail as the central jet initially penetrated the region with a velocity of $0.70 \mathrm{~m} / \mathrm{s}$ and subsequently detached from the inside surface of the thumbnail. Fig 8 shows the velocity field $1 \mathrm{~mm}$ below the flat housing surface. In the inflow region velocities reached $1.5 \mathrm{~m} / \mathrm{s}$, and the TSS level reached 1600 dynes $/ \mathrm{cm}^{2}$. In the outflow region the velocity vectors were skewed slightly. The peak velocity in this region was $1.3 \mathrm{~m} / \mathrm{s}$. Within the thumbnail, the jet remained skewed, with a peak velocity of $1.3 \mathrm{~m} / \mathrm{s}$ and TSS level reaching 830 dynes $/ \mathrm{cm}^{2}$ along the edges of the jet. A small recirculation region was apparent at the upper boundary of the jet, and a much larger recirculation region was seen in the lower portion of the thumbnail figure. A similar behavior was observed at the 3-mm elevation.

During mid-systole, the velocities and TSS levels in the hinge inflow, outflow, and thumbnail regions were much lower than during either mid-acceleration or peak diastole. Velocities ranged from $0.25 \mathrm{~m} / \mathrm{s}$ to $0.55 \mathrm{~m} / \mathrm{s}$, and the TSS levels were generally less than 250 dyne $/ \mathrm{cm}^{2}$.

SJM 17-mm Regent hinge flow fields. Fig 9 shows the velocity field inside the $17-\mathrm{mm}$ Regent hinge at mid-acceleration, level with the flat housing surface. During this phase, the velocity vectors in the inflow hinge pocket were skewed toward the inside of the flow lumen. The velocities reached $0.50 \mathrm{~m} / \mathrm{s}$, and the TSS level reached 650 dynes $/ \mathrm{cm}^{2}$. Much less flow was concentrated in the outflow pocket during this phase. The mid-acceleration velocity fields were mostly stagnant at the remaining elevations.

At peak diastole, the velocity field at the level of the flat housing surface was similar to that at the midacceleration phase; the velocities reached $0.60 \mathrm{~m} / \mathrm{s}$, and the TSS level reached 800 dynes $/ \mathrm{cm}^{2}$. The peak diastolic velocity fields were mostly stagnant at the remaining elevations.

Fig 10 shows the velocity field at mid-systole, level with the flat housing surface. In the outflow hinge pocket the vectors flowed along the inside surface of 


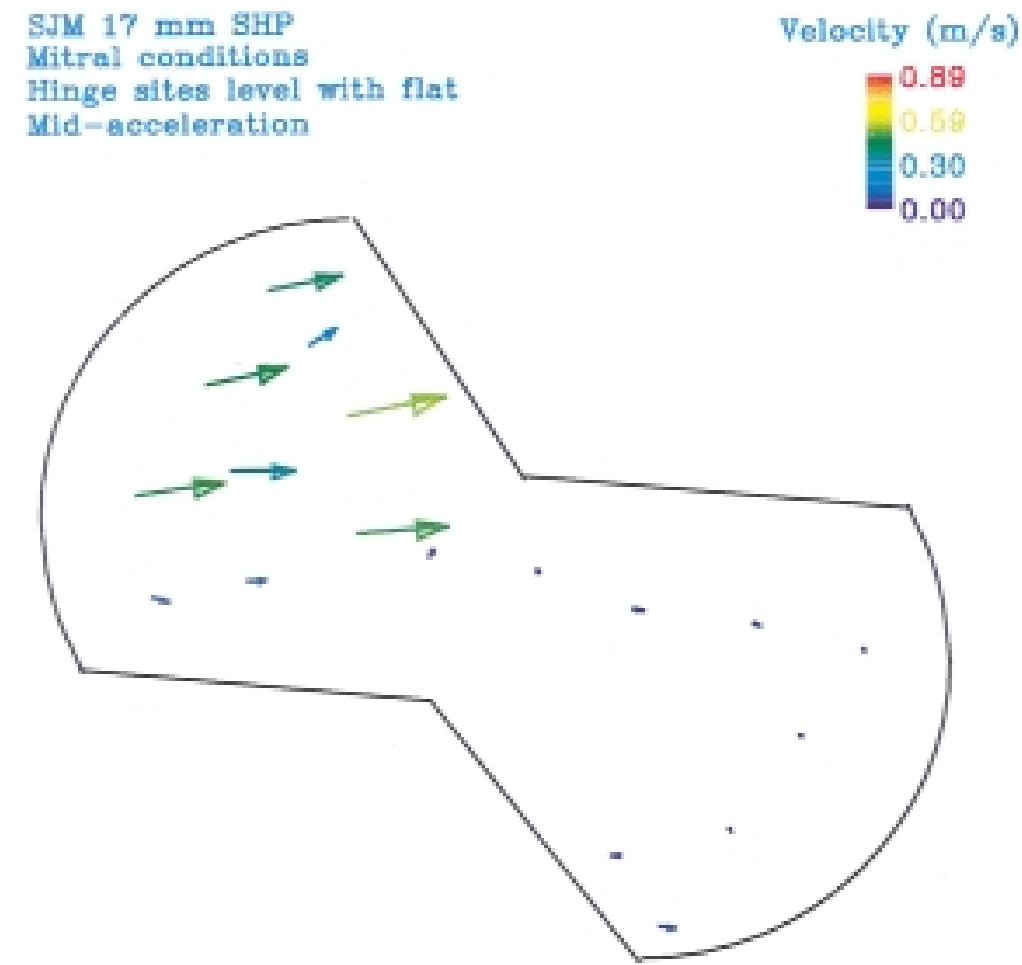

Fig 9. SJM 17-mm Regent hinge flow field at mid-acceleration, level with flat housing surface.

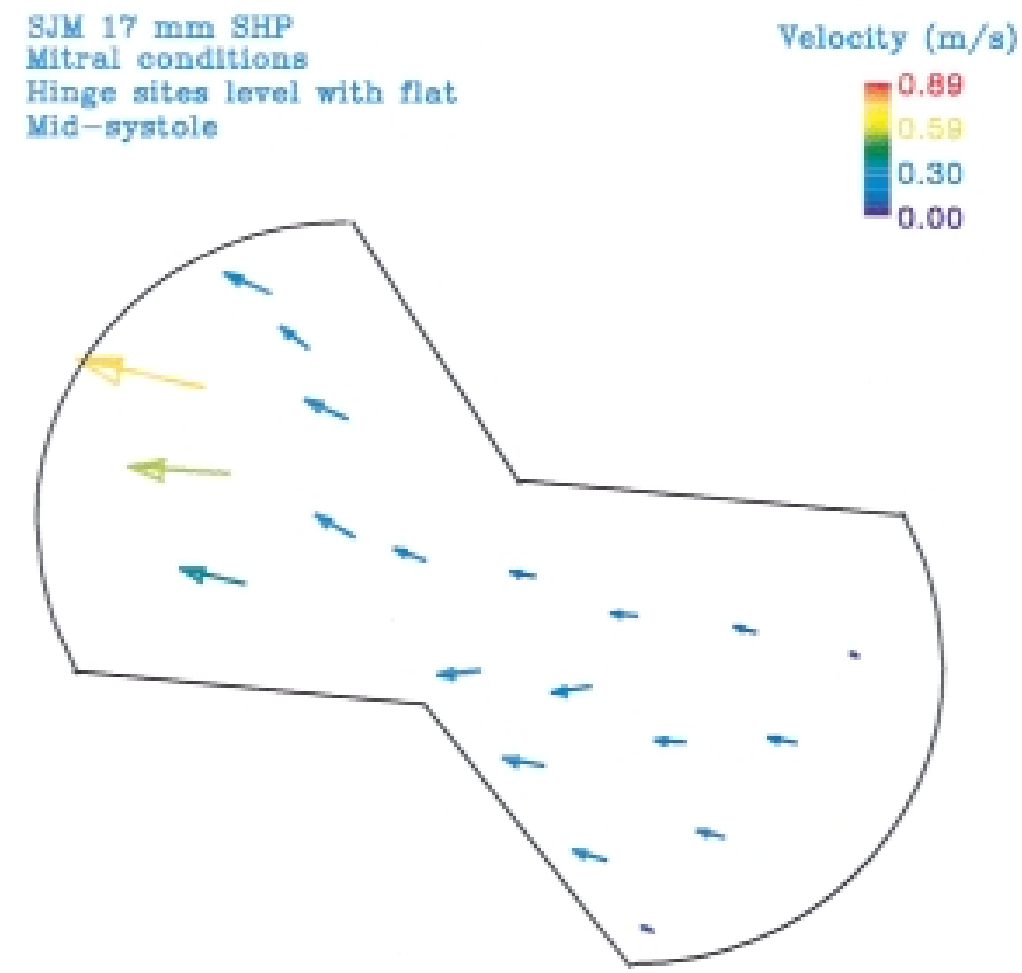

Fig 10. SJM 17-mm Regent hinge flow field at mid-systole, level with flat housing surface. 

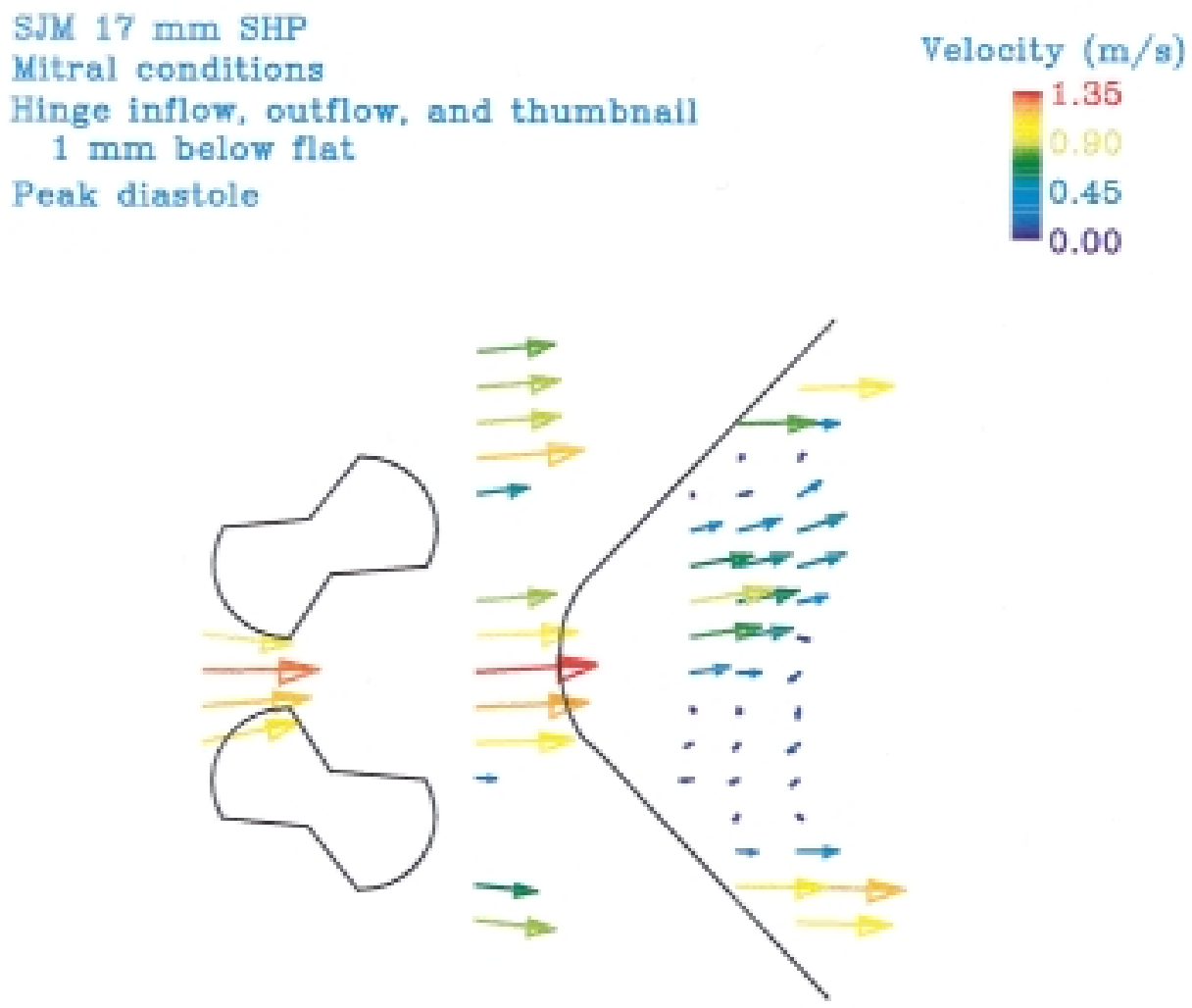

Fig 11. SJM 17-mm Regent hinge inflow, outflow, and thumbnail flow field at peak diastole, $1 \mathrm{~mm}$ below flat housing surface.

the leaflet ear, with velocities reaching $0.30 \mathrm{~m} / \mathrm{s}$. In the inflow hinge pocket the leakage jet flowed over the leaflet ear and out through the inflow side of the hinge. Velocities reached $0.75 \mathrm{~m} / \mathrm{s}$, and the TSS level reached 2000 dynes $/ \mathrm{cm}^{2}$ in this region. The mid-systolic velocity fields at the remaining elevations were qualitatively similar to those observed at the level of the flat housing surface, although with a lower leakage velocity range $(0.18 \mathrm{~m} / \mathrm{s}$ to $0.32 \mathrm{~m} / \mathrm{s})$.

SJM 17-mm Regent hinge inflow, outflow, and thumbnail flow fields. At mid-acceleration, the central jet was skewed at the $500-\mu \mathrm{m}$ and $1-\mathrm{mm}$ elevations, with recirculation regions apparent at the jet boundaries. At the 3-mm elevation, the central jet was axially dominant and penetrated more of the thumbnail region than it did at the other elevations. The peak jet velocity was $0.85 \mathrm{~m} / \mathrm{s}$, as measured at the 1-mm elevation.

At peak diastole, the thumbnail jet remained skewed at the $500-\mu \mathrm{m}$ elevation. The velocities in the jet reached $0.70 \mathrm{~m} / \mathrm{s}$. Fig 11 shows the velocity field along the hinge inflow and outflow regions and within the thumbnail, $1 \mathrm{~mm}$ below the flat housing surface. Velocities reached $1.4 \mathrm{~m} / \mathrm{s}$, and the TSS level reached
75 dynes $/ \mathrm{cm}^{2}$ in the inflow region between the open leaflets. In the outflow region between the two leaflets, the velocity vectors were skewed toward the upper portion of the figure. The velocities in the center of the outflow region ranged from $1.2 \mathrm{~m} / \mathrm{s}$ to $1.4 \mathrm{~m} / \mathrm{s}$, and the TSS level reached 400 dynes $/ \mathrm{cm}^{2}$ near the inside surfaces of the leaflets in the outflow region. Within the thumbnail, the jet continued to skew toward the upper edge of the figure. The peak velocity in the jet was 0.85 $\mathrm{m} / \mathrm{s}$, and the TSS level reached 970 dynes $/ \mathrm{cm}^{2}$ near the center of the jet. A small recirculation region formed near the upper edge of the thumbnail figure, whereas a larger recirculation region was apparent at the lower edge. Higher velocity flow, ranging from $0.85 \mathrm{~m} / \mathrm{s}$ to $1.4 \mathrm{~m} / \mathrm{s}$, was present near the edges of the thumbnail. The velocity field at the 3-mm elevation was similar to that at the 1-mm elevation, although with a more axially dominant outflow between the leaflets.

During mid-systole, the velocities and TSS levels in the 17-mm Regent hinge inflow, outflow, and thumbnail regions were lower than during either mid-acceleration or peak diastole, a pattern also observed in the 17$\mathrm{mm}$ HP flow fields. Velocities ranged from $0.20 \mathrm{~m} / \mathrm{s}$ to 


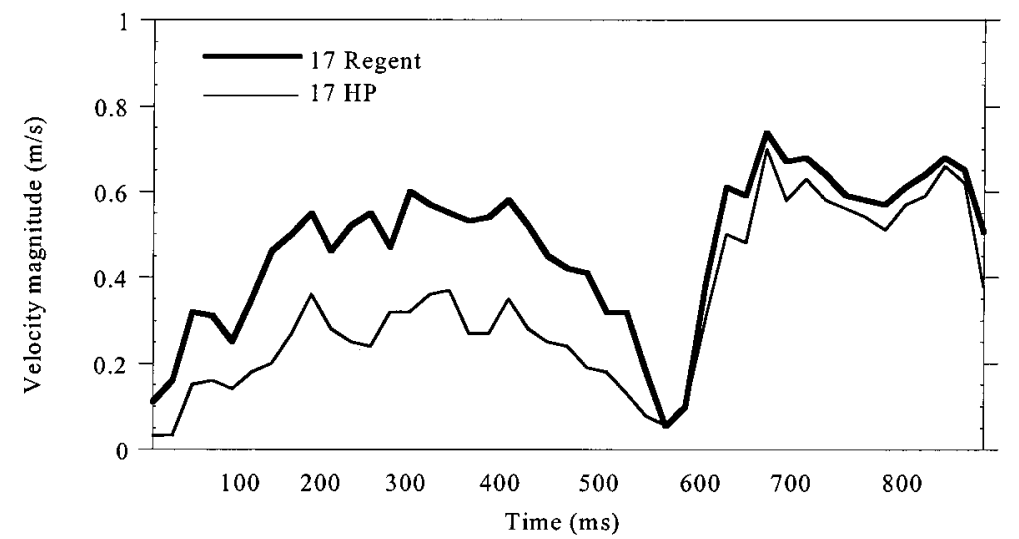

Fig 12. Temporal behavior of peak velocity magnitudes at the level of the flat housing surface within 17-mm HP and 17-mm Regent hinges.

Table I. Peak and time-averaged TSS levels in 17-mm HP and 17-mm Regent hinges

\begin{tabular}{lcccc}
\hline & & \multicolumn{2}{c}{ Percentage of hinge sites with peak TSS level } \\
\cline { 3 - 5 } Valve & $\begin{array}{c}\text { Time-averaged } \\
\text { systolic TSS level }\left(\text { dynes } / \mathrm{cm}^{2}\right)\end{array}$ & $\begin{array}{c}\text { Peak TSS level } \\
\left(\text { dynes } / \mathrm{cm}^{2}\right)\end{array}$ & $\begin{array}{c}\text { Level } \\
\text { Level }\end{array}$ & $\geq 1000$ dynes $/ \mathrm{cm}^{2}$ \\
\hline 17 -mm HP & 420 & 2900 & 33 & 19 \\
17 -mm Regent & 720 & 2000 & 22 & 12 \\
\hline
\end{tabular}

$0.40 \mathrm{~m} / \mathrm{s}$, and the TSS levels were generally less than 400 dynes $/ \mathrm{cm}^{2}$.

\section{Discussion}

These studies have provided, for the first time, an understanding of the flow structures created in the SJM hinge region under physiologic conditions. The results of these studies show that even within the confines of the hinge mechanism, the flow fields are very complex and unsteady. Table I summarizes the largest peak systolic and time-averaged TSS levels measured in the hinge regions of the 17-mm HP and 17-mm Regent valves.

General features of SJM hinge flow. The flow fields within the hinge regions of these SJM valves were qualitatively dynamic. During systole, the highest leakage jet velocities and TSS levels tended to occur in the regions in which the leaflet impacted the hinge walls during valve closure. This action created a "door-slamming" effect, which, in the in vivo case, may serve to keep the hinge region cleared of any deposited blood elements or pooled coagulative factors. The active leaflet motion through the SJM hinge may thus be a contributing factor to its successful clinical performance.

These studies also suggest that the actual shape of the hinge mechanism influences the levels of turbulence, unsteadiness, or both generated inside the hinge region, which is in essence the origin of the leakage jet. For example, the peak TSS level $\left(>6000\right.$ dynes $\left./ \mathrm{cm}^{2}\right)$ previously measured in the MP hinge region ${ }^{3}$ was over twice as high as the TSS levels measured in either of the SJM hinges in the present study. One possible explanation for the higher TSS levels in the MP hinge is the presence of the sharp corners and angled inflow and outflow ramps in its hinge recess. Because a leakage jet flows through these abrupt changes in geometry in the MP hinge, there is a strong likelihood of jet detachment, direct impact, or both against the corner of the inflow ramp, both of which would lead to the formation of stagnation sites and possible regions of turbulence. In contrast, the streamlined, curved profile of the SJM hinge recess provides a more gradual change in geometry. Correspondingly, a leakage jet flowing through the SJM hinge design is more likely to follow the contour of the inside surface, resulting in fewer regions of flow separation and turbulence.

As a general note, these studies illustrate that the design of the hinge and the near-hinge regions dictates the overall features of the flow fields. However, as discussed below, a perhaps more nonintuitive finding of this work is that even small variations in otherwise identical designs can have important consequences in how the resulting flow fields are generated. 
Hinge and near-hinge flow regions of SJM 17-mm HP and 17-mm Regent valves. In this discussion comparisons of the HP and Regent flow fields were made at selected regions at similar locations, level with the flat housing surface of both hinges and also $1 \mathrm{~mm}$ below the flat housing surface within the thumbnail flow fields. Fig 12 shows the temporal behavior of the peak velocities at the level of the flat housing surface within the two hinge regions. Both traces show a similar temporal development during diastole, with the diastolic velocities in the 17-mm Regent and 17-mm HP hinges differing only by $0.25 \mathrm{~m} / \mathrm{s}$ or less. The HP and Regent systolic traces were virtually identical.

The flow field at the mid-acceleration phase of diastole in the 17-mm Regent hinge showed the possible beginning formation of a rotating structure, whereas the corresponding flow field in the $17-\mathrm{mm}$ HP hinge showed a more distinct rotating structure. During diastole, the slightly higher velocities in the 17 -mm Regent hinge contributed to slightly larger TSS levels than those measured in the 17-mm HP hinge. However, the higher forward flow velocities in the 17-mm Regent hinge may lead to more improved forward flow washout of the hinge pockets compared with that of the 17-mm HP hinge. The flow fields at mid-systole showed that for both valve designs, the highest velocity leakage flow was detected through the inflow hinge pocket. In the regions of comparison at the level of the flat housing surface, the highest leakage jet velocity and TSS level in the $17-\mathrm{mm} \mathrm{HP}$ hinge were $0.70 \mathrm{~m} / \mathrm{s}$ and 1000 dynes $/ \mathrm{cm}^{2}$, respectively, whereas within the $17-\mathrm{mm}$ Regent hinge, the highest leakage jet velocity and TSS level were $0.75 \mathrm{~m} / \mathrm{s}$ and 1300 dynes $/ \mathrm{cm}^{2}$, respectively. The temporal development of the velocities and TSS levels, the peaks of these values, and the duration over which the peaks were sustained were similar for the two valve designs. These results suggest that the hinge flow dynamics of the 17-mm Regent are at least equivalent to, and possibly superior to, those of the 17-mm HP.

The physical structure of the central jets in the 17mm Regent and 17-mm HP thumbnails was very similar during the mid-acceleration phase of diastole, during peak diastole, and throughout most of the forward flow phase thereafter. Both flow fields showed preferential skewing of the jets. Inside the central (diastolic) jets, velocities approached $1.0 \mathrm{~m} / \mathrm{s}$ in the $17-\mathrm{mm}$ Regent and $1.4 \mathrm{~m} / \mathrm{s}$ in the $17-\mathrm{mm}$ HP; the TSS level reached 800 dynes $/ \mathrm{cm}^{2}$ in the $17-\mathrm{mm}$ Regent and 600 dynes $/ \mathrm{cm}^{2}$ in the $17-\mathrm{mm}$ HP. During systole, the peak velocity was approximately $0.40 \mathrm{~m} / \mathrm{s}$ for both thumbnail regions, and the peak TSS level was approximately 100 dynes $/ \mathrm{cm}^{2}$ for both thumbnail regions. The tempo- ral development of the velocities and TSS levels, the peaks of these values, and the duration over which the peaks were sustained were similar for the two thumbnail regions. Thus, quantitatively, the flow fields through the two thumbnail regions were similar. These results suggest that the thumbnail flow dynamics of the 17-mm Regent are equivalent to those of the 17-mm HP.

\section{Conclusions}

The LDV measurements conducted in the hinge and near-hinge flow regions of the SJM valves provided a description of the flow fields in this hinge design and allowed for a direct comparison between the hinge flow dynamics of the HP and Regent series. Similar peak flow velocities and qualitatively similar flow features were observed in the two hinges. The active leaflet motion through the SJM hinge and its streamlined hinge profile are important design features that may contribute to the low incidence of thromboembolism of this valve design. On the basis of the findings of this study and the consistency of the two valve designs, the hinge flow characteristics of the Regent series should be superior to those of the HP series.

\section{Limitations}

The LDV measurements were conducted at only selected locations and thus do not completely define the flow fields within the hinge regions of the valves. Because of optical access limitations, only 2-dimensional velocity measurements were obtained. It is possible that these confined flow fields may indeed be 3dimensional because of the complex geometries formed by the mating of the leaflet and hinge. Other experimental techniques, such as high-speed flow visualization or the use of scaled-up models, ${ }^{5,12}$ may be useful in assessing the flow fields more completely. However, the conclusions of the present study are unlikely to be affected. These findings are intended to be viewed as supplementary data that should be considered together with other performance criteria, such as satisfactory and well-established structural durability and clinical histories.

\section{REFERENCES}

1. Black M, Drury P. Mechanical and other problems of artificial valves. In: Berry $\mathrm{C}$, editor. Current topics in pathology. Berlin: Springer-Verlag; 1993. p. 128-59.

2. Vallana F, Rinaldi S, Galetti P, Nguyen A, Piwnica A. Pivot design in bileaflet valves. ASAIO J 1992;38:M600-6.

3. Ellis J, Healy T, Fontaine A, Saxena R, Yoganathan A. Velocity measurements and flow patterns within the hinge region of a Medtronic parallel bileaflet mechanical heart valve with clear housing. J Heart Valve Dis 1996;5:591-9.

4. Gross J, Shu M, Dai F, Ellis J, Yoganathan A. A microstructural 
flow analysis within a bileaflet mechanical heart valve hinge. J Heart Valve Dis 1996;5:581-90.

5. Healy T, Fontaine A, Ellis J, Walton S, Yoganathan A. Visualization of the hinge flow in a 5:1 scaled model of the Medtronic parallel bileaflet heart valve prosthesis. Exp Fluids 1998;25:512-8.

6. Baudet E, Puel V, McBride J, Grimaud J, Roques F, Clerc F, et al. Long-term results of valve replacement with the St. Jude Medical prosthesis. J Thorac Cardiovasc Surg 1995;109:858-70.

7. Arom K, Emery R, Petersen B, Radosevich D. St. Jude Medical valve prosthesis: health status of the patient after 15 years. Ann Thorac Cardiovasc Surg 1996;2:43-9.

8. Williams A. Shear-induced fragmentation of human erythrocytes. Biorheology 1972;10:303-11.
9. Wurzinger L, Opitz R, Wolf M, Schmid-Schonbein H. Shearinduced platelet activation: a critical reappraisal. Biorheology 1985;22:399-413.

10. Baldwin J, Deutsch S, Geselowitz D, Tarbell J. Estimation of Reynolds stresses within the Penn State left ventricular assist device. ASAIO Trans 1990;36:M274-8.

11. Giersiepen M, Wurzinger L, Opitz R, Reul H. Estimation of shear stress-related blood damage in heart valve prostheses-in vitro comparison of 25 aortic valves. Int J Artif Organs 1990;13:3006.

12. Gao Z, Hosein N, Dai F, Hwang N. Pressure and flow fields in the hinge region of bileaflet mechanical heart valves. J Heart Valve Dis 1999;8:197-205. 